\title{
A Macroscopic Model of an Aggregation Experiment using Embodied Agents in Groups of Time-Varying Sizes
}

\author{
William Agassounon, Alcherio Martinoli \\ California Institute of Technology \\ Collective Robotics Research Group \\ Pasadena, CA, 91125 , USA \\ \{agassw, alcherio\}@caltech.edu
}

\begin{abstract}
In this paper, we present a mathematical model of an aggregation experiment carried out using multiple embodied agents in teams of time-varying sizes. The aggregation experiment is concerned with the gathering and the clustering of small objects initially scattered in an enclosed arena. The number of active agents engaged in the aggregation task is varying according to a local, distributed stimulus-response law, similar to the behavior observed in ant colonies. We use a set of differential equations to describe the dynamics of the system at the macroscopic level. We validate the predictions of this model by comparing them to experimental data obtained using a sensor-based embodied simulator. Results show that the proposed approach delivers accurate predictions and constitutes a computationally efficient tool for studying aggregation experiments with groups of constant or variable sizes. The simplicity of the model suggests that it is easily applicable to other aggregation or segregation experiments characterized by different agent capabilities and individual control algorithms.
\end{abstract}

Keywords: multi-agent systems, division of labor aggregation, embodied simulations, self-organization.

\section{INTRODUCTION}

There are two main approaches to control in multiagent systems, one centralized and the other distributed. This paper contributes to research in multiple embodied agent systems based on the latter by proposing a mathematical approach for studying such complex systems. Swarm Intelligence [3] is a new behavioral and control paradigm in the design of multi-agent systems. It is characterized by the use of large numbers of autonomous agents to accomplish tasks while relying solely on the collaborative behavior that emerges from interactions among agents and between individuals and the environment.

To understand complex biological colonies in general and study their inspired applications in multi-robot systems, few accurate and computationally efficient microscopic or macroscopic models have been proposed. While the former describe the individual's interactions with its teammates and the environment (see for instance $[7,8]$ ), the latter offer a direct description of the collective group behavior. Among the macroscopic models developed so far, some focus on task allocation mechanisms $[1,3,12,13\}$. others offer a reliable but mostly qualitatively accurate theoretical paradigm [6]. In general, the macroscopic models are more computationally efficient than their microscopic counterparts even if the latter often offer more detailed information about the dynamics of the same systems they are applied to.

In the following, we present a mathematical model applied to a distributed, multi-agent manipulation experiment concerned with cluster formation. This approach accurately predicts the quantitative outcome of different variables of the experiment. The proposed model completes and generalizes a first macroscopic model based on difference equations that we introduced in [1]. Finally, it has the additional advantage of being easily applicable to other distributed manipulation experiments such as puck clustering [4] and object sorting [9].

In the next section, we present the case study, the simulation tool used as test-bed, and the object clustering algorithm In section III we introduce and apply our methodology to the system. The results and discussions are in section IV and the concluding remarks are in section $\mathrm{V}$. 


\section{A Case Study: The Aggregation Experiment}

The aggregation or cluster formation experiment by multiple autonomous agents is an excellent example of distributed problem solving. It can be roughly described as follows: several objects present in a given arena are to be sorted and gathered in clusters by some autonomous agents $[4,7,9]$. The particular case study we used in this paper is concerned with the use of a team of 10 autonomous agents picking up and clustering 20 small objects (referred to as "seeds") scattered in an enclosed $80 \times 80 \mathrm{~cm}$ arena (see $[1,7])$.

\section{The Sensor-based Simulator}

We implemented the aggregation experiment in Webots 2.0.1, a 3D sensor-based, kinematics simulator [10] of Khepera robots [11]. The simulator computes trajectories and sensory inputs of the embodied agents in an arena corresponding to the physical set-up (for examples see Figures 1 and 2).

The environment consists of an $80 \times 80 \mathrm{~cm}$ arena where twenty small seeds are randomly scattered at the beginning of the experiment. The average speed ratio for this experiment with 10 robots between Webots and real time is about 7 on a PC Pentium III $800 \mathrm{MHz}$ workstation. When programmed in $\mathrm{C}$ and using Matlab to process the data, the speed ratio of this macroscopic model on the same computer as that described above is about 3000 .

2. The Aggregation and the Worker Allocation Algorithms

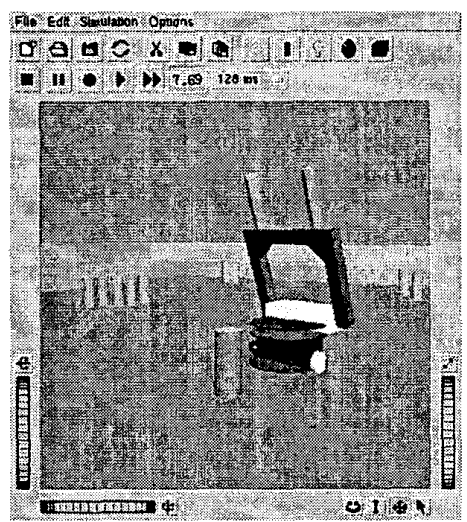

Figure 1: Close up of a simulated robot $5.5 \mathrm{~cm} \mathrm{in}$ diameter) in Webots equipped with a gripper turret in front of a seed.

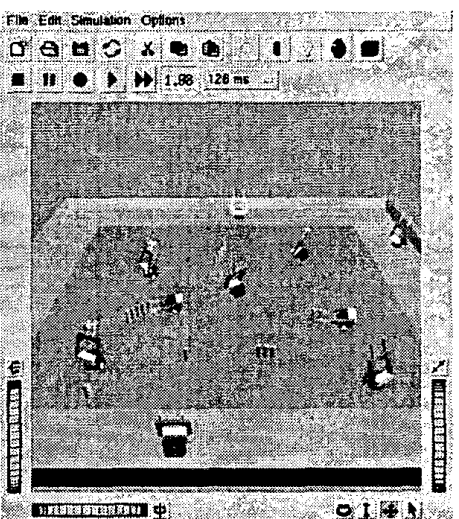

Figure 2: Experimental setup: inner area corresponds to the working zone and outer area is the resting zone. Aggregation in progress with 10 agents.

Each agent's behavior can be summarized by the following simple rules. In its default behavior, the agent moves straight forwards within the arena looking for seeds. When at least one of its six frontal proximity sensors is activated, the agent starts a discriminating procedure. Two cases can occur: if the agent is in front of a large object (a wall, another agent, or the body side of a cluster of seeds), the object is considered as an obstacle and the agent avoids it. In the second case, a small object is considered as a seed. If the agent is not carrying a seed, it grasps the seed with the gripper, otherwise, it drops the seed it is carrying close to that it has found; then, in both cases, the agent resumes looking for seeds. With this simple individual behavior, the team is able to gather objects in clusters of increasing size. A cluster is defined as a group of seeds whose neighboring elements are separated by at most one seed diameter. Note that, because agents identify only the two end seeds of a cluster as seeds (as opposed to obstacles), clusters are built in lines.

The embodied agents described in section II are not endowed with the physical capability of knowing when the aggregation task is finished e.g., they do not have a global perception of the environment. However, each of these agents is able to estimate the amount of time it spends searching for seeds and relate that individual information to the availability of work. This is at the core of our current worker allocation mechanism described as follows. When an agent has not been able to work (i.e. to pick up and drop a seed) for a reasonable amount of time that depends on the experimental setup, its propensity to accomplish the task is decreased. If the amount of time spent in the search for work to accomplish is above a given fixed threshold (i.e. a $T_{s}$ time-out), a first deterministic 
switching mechanism prompts the agent to leave the working zone and rest in the adjacent parking space. An agent carrying a seed that decides to become inactive cannot do so until it finds an appropriate spot (i.e. one tip of a cluster) to drop the seed. A second deterministic switching mechanism could allow the agent to resume the working activity as soon as the resting time has exceeded a $T_{r}$ time-out. This is not necessary if the demand does not increase at any time, which is the case with the present aggregation experiment.

Thus, with this simple algorithm with two thresholds common to all the teammates, the agents are able to locally evaluate the aggregation demand and to decide whether to work or rest with no need of a central controller (see $[1,2]$ for more details). This task allocation mechanism is similar to that observed in some ant colonies $[5,14]$ where it has been shown that an individual performs a task as long as the level of the demand stimulus of the task e.g., a pheromone, exceeds its threshold for that particular task $[3,13]$.

\section{The Mathematical Model}

The dynamical variables of this model are $n_{k}(t)$, the number of clusters of size $k, x_{c}(t)$, the fraction of agents carrying a seed, and $x_{s}(t)$, the fraction of agents searching for a seed to pick up respectively. In the following, we describe the quantitative dynamics of the system through a set of differential equations.

\section{Dynamics of the Manipulated Objects}

In subsections 1.1 and 1.2 , we present the quantitative dynamics of the clusters of seeds and estimate the average cluster size at steady state.

\section{I. Dynamics of the Clusters of Seeds}

The general rate equation for any given cluster of size $k$ is described by

$$
\begin{aligned}
\frac{1}{W} \frac{d n_{k}}{d t} & =\left(\gamma_{k+1}^{d e c} n_{k+1}(t-\tau)-\gamma_{k}^{d e c} n_{k}(t-\tau)\right) x_{s}(t-\tau) \\
& +\left(\gamma_{k-1}^{i n c} n_{k-1}(t-\tau)-\gamma_{k}^{i n c} n_{k}(t-\tau)\right) x_{c}(t-\tau)
\end{aligned}
$$

where $W$ represents the total number of agents and $\tau$ the average amount of time an agent needs to pick up/drop a seed. Finally, $\gamma_{k}^{d e c}$ and $\gamma_{k}^{i n c}$ corresponds to the rate at which an agent encounters and picks up a seed from a cluster of size $k$ and drops a seed next to a cluster of the same size respectively, if such a cluster is present in the arena. It is worth noting that the last two parameters are both design and experimental setup related parameters as they depend on the area occupied by a cluster related to the total area of the environment in which the agents are moving and the density of obstacles. For instance, if the working area surface is simply reduced, the team of agents will encounter clusters and obstacles at a higher rate in the new environment than in the previous one. Similarly, a larger $\tau$ will result in a slower aggregation rate, as agents will spend longer time to modify clusters.

On the right hand side of equation 1, the first term corresponds to the contribution of the loaded agents to the dynamics. These agents can increase or decrease the number of clusters of size $k$ by dropping a seed next to one of size $k-l$ or $k$. Similarly, the second term translates the contribution of agents that are searching for seeds to pick up.

1.2. Steady State Analysis

As shown by Martinoli et al. [7,8], if the agents do not withdraw (i.e. the team size is constant) and do not drop a seed unless it is next to another seed or pick up an internal seed of a cluster, the number of clusters monotonically decreases and eventually a single cluster always arises. Based on the assumption above, we studied the steady state of the system after a single cluster arises. In that state the average number of seeds on the arena surface will remain constant. We found that the average size of the single cluster in steady state (denoted $\tilde{n}^{\infty}$ ) for a fixed team size $W$ is given by:

$$
\tilde{n}=M-\frac{1}{1+\rho} W
$$

where $M$ corresponds to the total number of seeds in the environment, thus the size of the largest cluster possible and $\rho=\gamma_{k}^{i n c} / \gamma_{k}^{\text {dec }}$ is a constant for all $k<M$. A more practical way of drining $\tilde{n}^{\infty}$ is that it represents the average size of the unique cluster remaining in the environment as some seeds are continuously being picked up from and dropped at its two end tips.

\section{Dynamics of the Active Agents}

Following prior work on the group size of active workers in multi-agent systems $[12,13]$, we present the variation of the fraction of agents instead of the number of agents, as this is better suited for generalization and scalability purposes. Equation 3 represents the dynamics of the fraction of agents searching for seeds to pick up. In that equation $x(t)$. represents the fraction of agents that withdraw from the task at time $t$. On the right hand side of equation 3 the first term corresponds to the variation due to agents that drop seeds and start searching for a new one to pick up and agents that stop searching for seeds to pick up one they have just found. A similar equation gives the dynamics of the fraction of loaded agents.

$$
\begin{aligned}
\frac{d x_{s}}{d t}= & \sum_{k=1}^{M}\left(\gamma_{k}^{i n c} n_{k}(t-\tau) x_{c}(t-\tau)-\gamma_{k}^{d i c} n_{k}(t) x_{s}(t)\right) \\
& +\frac{d x}{d t}
\end{aligned}
$$


We introduce a new approach for estimating $x(t)$ based on the manipulation stimulus $S(t)$ associated with the aggregation task. In this case study, $S(t)$ is expressed in time units and represents the average amount of time agents would spend to find a seed to manipulate at time $t$. There are similarities and differences between the stimulus as it is defined for this aggregation task and the demand stimulus associated with a task carried out in an ant colony. As a global information, $S(t)$ is similar to the pheromone level present in the ant nest that regulates the foraging activity of the colony. However, while in the ant nest the stimulus is an increasing function of the demand, in our experiment the higher is the aggregation demand, the lower is the manipulation stimulus since this does not corresponds to a pheromone concentration but to the time spent before finding a seed to manipulate. Furthermore, the distributed nature of our aggregation experiment and the absence of explicit communication mechanisms among agents, generate differences in the local demand estimations. We capture these differences by introducing a noise source in the manipulation stimulus estimated at the individual level i.e. $\hat{S}(t)$. In other words, even if all the individuals have the same activity-threshold $T_{S}$ (homogeneous team), conversely to the common pheromone level permeating the nest of an ant colony, our embodied agents do not perceive the same manipulation stimulus due to the use of their local perception to evaluate it. Hence for a homogeneous team of agents, we can express the individual estimation of this demand stimulus and the fraction of active agents by (4) and (6) respectively.

$$
\begin{aligned}
& \hat{S}(t)=S(t)+\varepsilon(t) \\
& \frac{d S}{d t}=\lambda_{1} \frac{d c}{d t}+\lambda_{2} \frac{d w}{d t} \\
& x(t+\delta t)=x(t) \Gamma(t)
\end{aligned}
$$

$\varepsilon(t)$ represents a white, additive noise. For this aggregation experiment, we relate the variation of $S(t)$ to the availability of manipulation sites and the number of agents present in the arena at time $t$ as described in equation 5 . In that equation, $c(t)$ and $w(t)$ represent the average cluster size and the average number of agents present in the arena at time $t$ respectively. $\lambda_{1}$ and $\lambda_{2}$ represent the unit increase/decrease of the average amount of time that an agent needs to find and manipulate a seed present in the arena due to a unit increase/decrease of the average cluster size and the average number of agents respectively. Note that $\lambda_{I}$ is in time units per seed and $\lambda_{2}$ is in time units per agent. More practically as the aggregation process goes on, $S(t)$ translates the increasing scarcity of available seeds to manipulate and also captures the change in the agent-to-agent interference rate as the number of agents decreases over time. As a comparison, this increasing scarcity of seeds to manipulate over time is equivalent to the chemical feedback that ants rely on to estimate the progress in task performance $[5,13,14]$.

In equation $6, \Gamma(t)$ is the probability that in response to the stimulus an agent remains active at time $t$ and $\delta t$ translates how fast an agent can change from active to inactive state. For instance, in this cluster formation experiment, an agent needs about 10 seconds to pick up or drop a seed and travels at an average speed of 8 $\mathrm{cm} / \mathrm{sec}$. Therefore the average quantity of any constituent of the environment changes little over less than 10 seconds time. Finally, we use a white Gaussian noise of zero mean and variance $\sigma^{2}$ as an example. The probability $\Gamma(t)$ is then given by equation 7 where $\Theta($. is a step function that assures that $t>T_{s}$.

$$
\Gamma(t)=1-\frac{1}{\sigma \sqrt{2 \pi}} \int_{T,-S(t)}^{+\infty} \mathrm{e}^{-\frac{u^{2}}{2 \sigma^{2}}} d u \Theta\left(t-T_{s}\right)
$$

\section{RESULTS AND DISCUSSION}

In the following we present and compare results obtained using the proposed model with the embodied simulator presented above. Each aggregation run lasted 10 hours. We carried out 30 simulation runs using the embodied simulator. All error bars represent the standard deviations among runs. The mean time to pick up/drop a seed i.e. $\tau$ was measured from a same embodied agent. Then for any cluster of size $k$, we calculated $\gamma_{k}^{\text {dec }}$ and $\gamma_{k}^{\text {inc }}$ by multiplying the probability that an agent encounters such a cluster and picks up/drops a seed from/at its tips by the seed picking/dropping rate measured using the same procedure proposed in $[7,8]$. For the results presented below, we hand-coded: $T_{s}=25$ minutes, $\lambda_{l}=0.98$ minutes per seed, $\lambda_{2}=0.91$ minutes per agent, and $\sigma=4.83$ minutes. $T_{s}$ is a design choice (the same in the model and the embodied simulator) while $\lambda_{1}, \lambda_{2}$, and $\sigma$ are free parameters of the model.

\section{Aggregation without Worker Allocation}

Without worker allocation, the aggregation experiments are characterized by a constant team size, i.e. ten active agents from the beginning till the end of the experiment. Figure 3 presents the average size of the clusters over time. Using the $\gamma$-parameters calculated above, it comes that $\tilde{n}^{\infty} \approx 15.21$ seeds, and Figure 3 shows that after 10 hours into the aggregation process the average cluster size is close to 
this value for both the macroscopic model and the embodied simulator.

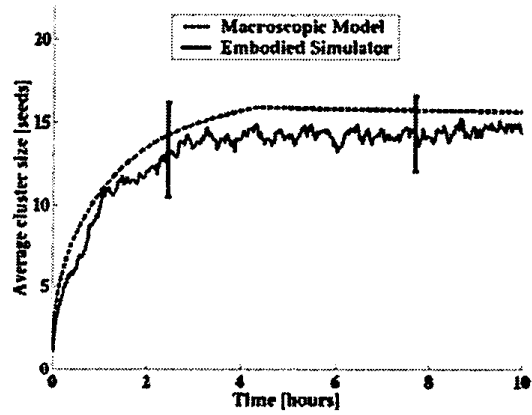

Figure 3: Aggregation experiment without worker allocation with 10 agents in an $80 X 80 \mathrm{~cm}$ arena.

Figure 3 shows a first phase when the average cluster size increases steadily from 1 seed to about 15 seeds and a second phase when the average cluster size remains on average constant around 15 seeds. This can be explained by the fact that, once a single cluster arises, only two manipulation sites remain in the environment (i.e. the two end tips of that cluster). Since the probabilities of picking up and dropping a seed are empirically very close (hence so are $\gamma_{k}^{\text {dec }}$ and $\gamma_{k}^{i n c}$ ), during that last phase of the aggregation process, at any given time, about half of the active workers will be carrying a seed and the remaining portion will not.

2. Aggregation with Worker Allocation

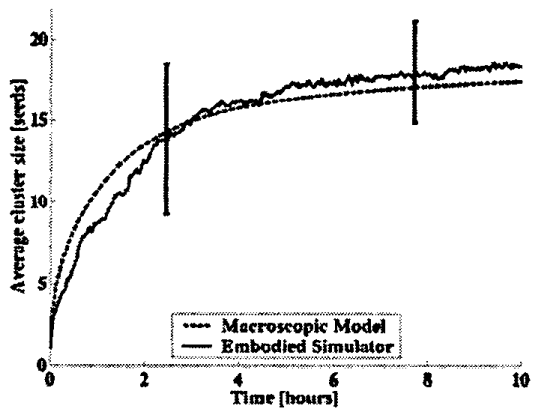

Figure 4: Aggregation experiment with worker allocation with 10 agents in an $80 \times 80 \mathrm{~cm}$ arena

Figures 4 and 5 show that conversely to the case without worker allocation, during the last phase of the aggregation, the average cluster size remains an increasing function of time eventually reaching 20 seeds, the optimal largest value possible, while the number of active workers in the environment decreases. As predicted in section IV.1, reducing the number of active agents, consequently increases the size of the single cluster during the last phase of aggregation.

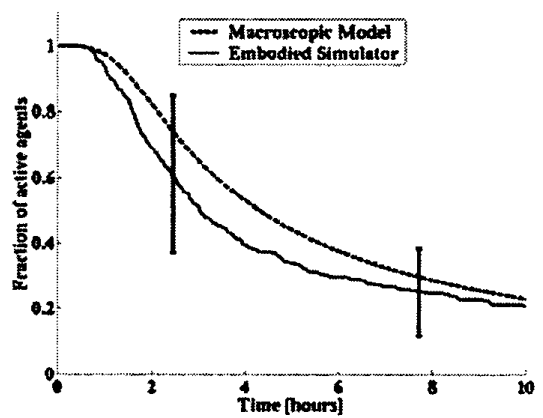

Figure 5: Fraction of active agents over time in an $80 \times 80 \mathrm{~cm}$ arena.

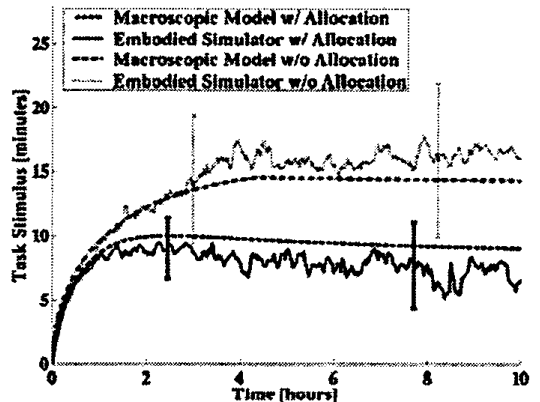

Figure 6: Evolution of the task stimulus with and without worker allocation

Figure 6 presents the evolution of the manipulation stimulus over time. The upper two curves represent the manipulation stimulus for the aggregation experiment without worker allocation while the lower curves were obtained using worker allocation. In both cases, the manipulation stimulus function presents two evolution phases. Without worker allocation, the stimulus increases from 0 to reach about 15 minutes during the first phase and this corresponds to the time when the number of manipulation sites decreases from 20 to 2 seeds. During the second phase the stimulus remains on average constant around 15 minutes due to the low number of manipulation sites (only two remain and it takes longer to find one) and the high agent-to-agent interference rate. With worker allocation, the manipulation stimulus remains on average smaller than 
in the previous case and slightly decreases during the second phase. This is explained by the fact that as there are fewer agents, the interference rate is considerably reduced and the remaining agents spend less time finding the remaining manipulation sites. The decrease in the demand suggests that agent-to-agent interference is predominant when the team size is large.

Finally, we obtain a good (quantitative) agreement between the macroscopic model and embodied simulations. Although this was achieved by using three free parameters, the significance of these parameters is intuitively clear and the model helps to shed light on the complex system dynamics resulting simultaneously from aggregation and worker allocation.

\section{CONCLUSION}

In this paper, we have presented a mathematical model for studying aggregation experiments using embodied agents in groups of fixed or variable sizes. We have validated the predictions of the macroscopic model with a realistic, microscopic, embodied simulator. Results show that the proposed approach delivers quantitatively accurate predictions and constitutes a computationally efficient tool. The simplicity of the model suggests that it is easily applicable to other aggregation or segregation experiments characterized by different agent capabilities and individual control algorithms. Future work will involve an effort to remove the free parameters, to generalize this model to a methodology, and to investigate different methods of analyzing at the macroscopic level, the noise due to agents' partial perception.

\section{ACKNOWLEDGMENT}

We would like to thank Dr Ian Kelly for his remarks. This work is supported in part by the TRW Foundation and the Caltech Center for Neuromorphic Systems Engineering as part of the NSF Engineering Research Center Program under grant EEC-9402726.

\section{REFERENCES}

[1] W. Agassounon, A. Martinoli, and R. Goodman, "A Scalable, Distributed Algorithm for Allocating Workers in Embedded Systems", Proc. of the IEEE
SMC'01 Conf., Tucson, Arizona, Oct. 2001, pp. 3367 3373.

[2] W. Agassounon, and A. Martinoli, "Efficiency and Robustness of Threshold-Based Distributed Allocation Algorithms in Multi-Agent Systems", Proc. of the First Int. Joint Conf. on Autonomous Agents and MultiAgent Systems, July 2002, Bologna, Italy. To appear.

[3] E. Bonabeau, G. Theraulaz, and J.-L. Deneubourg, "Fixed Response Thresholds and the Regulation of Division of Labour in Insect Societies", Bulletin of Mathematical Biology, Vol.60, 1998, pp.753-807.

[4] R. Becker, O. Holland, and J.-L. Deneubourg, "From Local Actions to Global Tasks: Stigmergy and Collective Robotics", Proc. of the fourth International Workshop on the Synthesis and Simulation of Living Systems, MIT Press, 1994.

[5] D. Gordon, "Ants at Work: How an Insect Society is Organized", The Free Press, 1999.

[6] K. Lerman, A. Galstyan, A. Martinoli, and A. J. Ijspeert, "A Macroscopic Analytical Model of Collaboration in Distributed Robotic Systems", Artificial Life. To appear. (O) MIT Press.

[7] A. Martinoli, A. Ijspeert, and F. Mondada "Understanding Collective Aggregation Mechanisms: From Probabilistic Modeling to Experiments with Real Robots", Robotics and Autonomous Systems, 29 (1999), pp.51-63.

[8] A. Martinoli, A. Ijspeert, and L.G. Gambardella, "A Probabilistic Model for Understanding and Comparing Collective Aggregation Mechanisms", Proc. of ECAL'99, Lausanne, Switzerland, Sept. 1999, pp. 575584.

[9] C. Melhuish, O. Holland, and S. Hoddell, "Collective Sorting and Segregation in Robots with Minimal Sensing", Proc. of the Sth Conference on Simulation of Adaptive Behavior, Zurich, Germany, 1998.

[10] O. Michel, "Webots: Symbiosis Between Virtual and Real Mobile Robots", Proc. of ICVW '98 Paris, France, 1998, pp. 254-263.

[11] F. Mondada, E. Franzi, and P. lenne, "Mobile Robot Miniaturization: A Tool for Investigation in Control Algorithms", Proc. of ISER '93, Kyoto, Japan, 1993, 501-513.

[12] S. W. Pacala, D. M. Gordon, and H. C. J. Godfray, "Effects of Social Group Size on Information Transfer and Task Allocation", Evolutionary Ecology, vol. 10, 1996, pp. 127-165.

[13] G. Theraulaz, E. Bonabeau, and J.-L. Deneubourg, "Response Threshold Reinforcement and Division of Labour in Insect Societies", Proc. of Royal Society of London, Serie B, 1998, pp. 327-332.

[14] E. O. Wilson, "The Relation Between Caste Ratios and Division of Labour in Ant Genus Pheidole (Hymenoptera: Formidacea)", Behav. Ecol. Sociobiol. 16 (1984), pp. 89-98. 